\title{
Ulam Stability for the Composition of Operators
}

\author{
Ana Maria Acu $1, *,+$ (D) and Ioan Raşa $2,+$ \\ 1 Department of Mathematics and Informatics, Lucian Blaga University of Sibiu, Str. Dr. I. Ratiu, No. 5-7, \\ 550012 Sibiu, Romania \\ 2 Department of Mathematics, Faculty of Automation and Computer Science, Technical University of \\ Cluj-Napoca, Str. Memorandumului, No. 28, 400114 Cluj-Napoca, Romania; ioan.rasa@math.utcluj.ro \\ * Correspondence: anamaria.acu@ulbsibiu.ro \\ + These authors contributed equally to this work.
}

Received: 21 May 2020; Accepted: 10 July 2020; Published: 13 July 2020

check for updates

\begin{abstract}
Working in the setting of Banach spaces, we give a simpler proof of a result concerning the Ulam stability of the composition of operators. Several applications are provided. Then, we give an example of a discrete semigroup with Ulam unstable members and an example of Ulam stable operators on a Banach space, such that their sum is not Ulam stable. Another example is concerned with a $C_{0}$-semigroup $\left(T_{t}\right)_{t \geq 0}$ of operators for which each $T_{t}$ is Ulam stable. We present an open problem concerning the Ulam stability of the members of the Bernstein $C_{0}$-semigroup. Two other possible problems are mentioned at the end of the paper.
\end{abstract}

Keywords: Ulam stability; composition of operators; closed linear subspaces; $C_{0}$-semigroups

\section{Introduction}

Let $E, F$ be normed spaces and $A: D(A) \subset E \rightarrow F$ a linear operator. Denote by $N(A), R(A)$, the kernel, respectively the range of $A$ :

$$
N(A):=\{x \in D(A) \mid A x=0\}, R(A):=\{A x \mid x \in D(A)\} .
$$

There are several equivalent definitions of the Ulam stability of $A$; see, e.g., [1] and the references therein. One of them is (see, e.g., [2]):

Definition 1. We say that $A$ is an Ulam stable operator if a constant $K \geq 0$ exists such that for each $x \in D(A)$, there exists $y \in N(A)$ with $\|x-y\| \leq K\|A x\|$. In this case, $K$ is called an Ulam constant for $A$.

Define:

$$
K_{A}:=\sup \left\{\frac{d(x, N(A))}{\|A x\|} \mid x \in D(A) \backslash N(A)\right\},
$$

where $d(x, N(A)):=\inf \{\|x-z\| \mid z \in N(A)\}$.

Proposition 1. A is Ulam stable if and only if $K_{A}<\infty$. If $A$ is Ulam stable, then:

$$
K_{A}=\inf \{K \mid K \text { is an Ulam constant for } A\} .
$$

Proof. Let $K$ be an Ulam constant for $A$ and $x \in D(A) \backslash N(A)$. Then, there exists $y \in N(A)$ with $\|x-y\| \leq K\|A x\|$. Therefore,

$$
d(x, N(A)) \leq\|x-y\| \leq K\|A x\|, \text { and so } K_{A} \leq K<\infty
$$


Conversely, suppose that $K_{A}<\infty$, and let $\varepsilon>0$. Let $x \in D(A) \backslash N(A)$. Then:

$$
d(x, N(A)) \leq K_{A}\|A x\|<\left(K_{A}+\varepsilon\right)\|A x\|,
$$

so that there exists $y \in N(A)$ with $\|x-y\| \leq\left(K_{A}+\varepsilon\right)\|A x\|$. If $x \in N(A)$, we can take $y=x$. Summing up, $K_{A}+\varepsilon$ is an Ulam constant for each $\varepsilon>0$, and so, $A$ is Ulam stable.

The above considerations show also that (1) is valid.

Concerning the following result, see, e.g., [3], Remark 18, p.47, and Ex. 2.14, p.51.

Proposition 2. Let $E, F$ be Banach spaces, and let $A: D(A) \subset E \rightarrow F$ be a closed operator. Then, the following assertions are equivalent:

(i) There exists $C \geq 0$ such that $d(x, N(A)) \leq C\|A x\|, x \in D(A)$;

(ii) $R(A)$ is closed.

Combining Propositions 1 and 2, we get the following:

Corollary 1. Let $E, F$ be Banach spaces, and let $A: D(A) \subset E \rightarrow F$ be a closed operator. Then, $A$ is Ulam stable if and only if $R(A)$ is closed.

Ulam stability theory is multifaceted. The above definitions and results will be used in the next sections, but much more information concerning the state-of-the-art can be found in $[1,4,5]$ and the references therein. Relevant publications concerning the Ulam stability for the composition of operators are [2] ([Chapter 2]), [4,6,7]; our paper is motivated by these existing results and, to a certain extent, is a continuation of them. To the best of our knowledge, problems concerning the Ulam stability of the operators forming a semigroup are addressed here for the first time.

The paper is organized as follows. Section 1 is devoted to a result from [6], concerning the Ulam stability of the composition of two operators acting between Fréchet spaces. Working in the setting of Banach spaces, we give a simplified proof; the resulting Theorem 1 generalizes the results from $[7,8]$. In Section 2, we provide several applications and then construct a discrete semigroup with Ulam unstable members and an example of Ulam stable operators on a Banach space such that their sum is not Ulam stable. Section 3 is concerned with a $C_{0}$-semigroup $\left(T_{t}\right)_{t \geq 0}$ of operators for which each $T_{t}$ is Ulam stable. Moreover, we present an open problem concerning the Ulam stability of the members of the Bernstein $C_{0}$-semigroup. Conclusions, further projects, and other two possible problems are mentioned in Section 4.

\section{Ulam Stability for the Composition of Operators}

The following theorem can be found, in the more general setting of Fréchet spaces, in [6]. Here, we work in the setting of Banach spaces, which allows us to give a simplified proof based on [3] ([Theorem 2.10, p. 37]).

Theorem 1. Let $X, Y, Z$ be Banach spaces, and let $S: X \rightarrow Y, T: Y \rightarrow Z$ be linear, bounded, Ulam stable operators. The following statements are equivalent:

$$
\begin{aligned}
& T S: X \rightarrow Z \text { is Ulam stable, } \\
& R(S)+N(T) \text { is closed in } Y .
\end{aligned}
$$

Proof. First, suppose that (3) holds, i.e., $R(S)+N(T)$ is closed in $Y$. Since $S$ is Ulam stable, Corollary 1 shows that $R(S)$ is closed. $N(T)$ is also closed, because $T$ is bounded. Now, Ref. [3] 
([Theorem 2.10, p. 37]) showed that a constant $C \geq 0$ exists such that for each $y \in R(S)+N(T)$ there exist $u \in R(S), v \in N(T)$ with:

$$
y=u+v,\|u\| \leq C\|y\|,\|v\| \leq C\|y\|
$$

Let:

$$
z \in X,\|T S z\| \leq 1 .
$$

Let $K_{t}, K_{s}$ be Ulam constants for $T$ and $S$, respectively.

Since $K_{t}$ is an Ulam constant for $T$, from (5), we infer that there exists $a \in N(T)$ such that:

$$
\|S z+a\| \leq K_{t} .
$$

Now, $S z+a \in R(S)+N(T)$. According to (4) and (6), there exist $u \in R(S), v \in N(T)$ such that:

$$
\begin{aligned}
& S z+a=u+v, \\
& \|u\| \leq C\|S z+a\| \leq C K_{t} .
\end{aligned}
$$

Let $x \in X$ with $S x=u$. From (8), it follows that $\|S x\| \leq C K_{t}$. By using the Ulam stability of $S$ (with constant $\left.K_{s}\right)$, we infer the existence of $r \in N(S)$ such that:

$$
\|r-x\| \leq C K_{t} K_{s} .
$$

Let $p:=z-x+r$. Then $S p=S(z-x)$. On the other hand, (7) yields $S z-u=v-a$. However, $u=S x$ and $a \in N(T)$ and $v \in N(T)$; we deduce that $S(z-x) \in N(T)$, and so, $S p \in N(T)$. Thus, TSp $=0$, i.e., $p \in N(T S)$. Moreover, according to (9), $\|z-p\|=\|x-r\| \leq C K_{t} K_{s}$.

To resume, starting from (5), we proved that there exists $p \in N(T S)$ with $\|z-p\| \leq C K_{t} K_{s}$. Due to the linearity of $T S$, this shows that it is Ulam stable (with Ulam constant $C K_{t} K_{s}$ ).

Conversely, suppose that TS is Ulam stable. Then, Corollary 1 shows that $R(T S)$ is closed in $Z$. It is easy to verify (see also [6]) that:

$$
R(S)+N(T)=T^{-1}(R(T S)) .
$$

Consequently, $R(S)+N(T)$ is closed in $Y$, and this concludes the proof.

Remark 1. Briefly speaking, the proof of Theorem 3 in [6] has a "topological" character: it uses neighborhoods. Our proof of Theorem 1 has a "metric" character; it allows us to emphasize the relationship between the Ulam constants of $T, S$, and TS.

Remark 2. Theorem 3 in [6] and, in particular, the above Theorem 1 improve the following results:

(1) ([7]) If $S$ is surjective, then TS is Ulam stable;

(2) ([8]) If $N(T) \subset R(S)$, then TS is Ulam stable.

More general results, in the setting of linear operators on linear spaces endowed with semigauges, can be found in [4] and [1] ([Chapter 2]).

\section{Applications}

Theorem 1 can be applied, in particular, in the following situations.

Corollary 2. Let $Y, Z$ be Banach spaces, $T: Y \rightarrow Z$ a linear, bounded, Ulam stable operator, and $X \subset Y a$ closed linear subspace. Then, $\left.T\right|_{X}$, the restriction of $T$ to $X$, is Ulam stable if and only if $X+N(T)$ is closed in $Y$. 
Proof. Let $S: X \rightarrow Y, S x=x, x \in X$. Then, $S$ is Ulam stable and $T S x=T x, x \in X$. Thus, $\left.T\right|_{X}=T S$ and $R(S)=X$. To conclude the proof, it suffices to apply Theorem 1 .

Corollary 3. Let $Y$ be a Banach space, and let $T: Y \rightarrow Y$ be a linear, bounded, Ulam stable operator. If $\operatorname{dim}(N(T))<\infty$, then $T^{k}$ is Ulam stable, $k \geq 1$.

Proof. It is well known (see, e.g., [9]) and easy to prove that for $A, B: Y \rightarrow Y$,

$$
\operatorname{dim} N(A B)=\operatorname{dim} N(B)+\operatorname{dim}(N(A) \cap R(B)) .
$$

In particular,

$$
\operatorname{dim} N\left(T^{2}\right) \leq 2 \operatorname{dim} N(T)
$$

and by induction,

$$
\operatorname{dim} N\left(T^{k}\right) \leq k \operatorname{dim} N(T), k \geq 1 .
$$

Now, $R(T)$ is closed, and $N(T)$ is finite-dimensional; it follows that $R(T)+N(T)$ is closed, and Theorem 1 shows that $T^{2}$ is Ulam stable. By induction, we find that $T^{k}$ is Ulam stable, $k \geq 1$.

Corollary 4. Under the hypotheses of Theorem 1 , suppose that $Z=Y$ and $\operatorname{dim} N(T)<\infty$. Then, $T^{k} S$ is Ulam stable for all $k \geq 1$.

Proof. According to (10), $N\left(T^{k}\right)$ is finite-dimensional. $S$ is Ulam stable; hence $R(S)$ is closed. It follows that $R(S)+N\left(T^{k}\right)$ is closed, and Theorem 1 shows that $T^{k} S$ is Ulam stable.

Example 1. Let $Y$ be a Banach space, $T: Y \rightarrow Y$ a compact linear operator, I the identity operator on $Y$, and $\lambda \neq 0$. It is known (see, e.g., [1] ([Chapter 2, Theorem 49])) that $T-\lambda I$ is Ulam stable. On the other hand, $\operatorname{dim}(N(T-\lambda I))<\infty$; see, e.g., [10] ([Theorem 8.3-3]).

Now, Corollary 3 shows that $(T-\lambda I)^{k}$ is Ulam stable for all $k \geq 1$. This conclusion can be obtained also from [10] ([Corollary 8.3-6]), according to which $R\left((T-\lambda I)^{k}\right)$ is closed for all $k \geq 1$.

Let $B(p, q):=\int_{0}^{1} t^{p-1}(1-t)^{q-1} d t, p, q>0$, be Euler's Beta function. In what follows, let $\alpha, \beta \geq-1$, and consider the Beta type operators $\mathcal{B}_{n}^{\alpha, \beta}: C[0,1] \rightarrow C[0,1], n \geq 1$, defined by (see [11] and the references therein):

Definition 2. For $f \in C[0,1]$ and $x \in[0,1]$, we define:

(i) in case $\alpha=\beta=-1$ :

$$
\mathcal{B}_{n}^{-1,-1}(f ; x)= \begin{cases}f(0), & x=0 \\ \frac{\int_{0}^{1} t^{n x-1}(1-t)^{n-n x-1} f(t) d t}{B(n x, n-n x)}, & 0<x<1 \\ f(1), & x=1\end{cases}
$$

(ii) in case $\alpha=-1, \beta>-1$ :

$$
\mathcal{B}_{n}^{-1, \beta}(f ; x)= \begin{cases}f(0), & x=0, \\ \frac{\int_{0}^{1} t^{n x-1}(1-t)^{n-n x+\beta} f(t) d t}{B(n x, n-n x+\beta+1)}, & 0<x \leq 1 ;\end{cases}
$$


(iii) in case $\alpha>-1, \beta=-1$ :

$$
\mathcal{B}_{n}^{\alpha,-1}(f ; x)= \begin{cases}\frac{\int_{0}^{1} t^{n x+\alpha}(1-t)^{n-n x-1} f(t) d t}{B(n x+\alpha+1, n-n x)}, & 0 \leq x<1, \\ f(1), & x=1 ;\end{cases}
$$

(iv) in case $\alpha, \beta>-1$ :

$$
\mathcal{B}_{n}^{\alpha, \beta}(f ; x)=\frac{\int_{0}^{1} t^{n x+\alpha}(1-t)^{n-n x+\beta} f(t) d t}{B(n x+\alpha+1, n-n x+\beta+1)}, 0 \leq x \leq 1
$$

Theorem 2. (i) For each $n \geq 1, \mathcal{B}_{n}^{\alpha, \beta}$ is not Ulam stable.

(ii) Consider the discrete semigroup $\left(\mathcal{B}_{n}^{\alpha, \beta}\right)_{j=0,1, \ldots}^{j}$. For $j=0$, one has the identity operator, which is Ulam stable. For each $j \geq 1,\left(\mathcal{B}_{n}^{\alpha, \beta}\right)^{j}$ is not Ulam stable.

Proof. $\mathcal{B}_{n}^{\alpha, \beta}$ is injective. For $\alpha=\beta=-1$, respectively $\alpha=\beta=0$, proofs can be found in [12], respectively [8]. The proof in the general case is similar, and we omit it.

For each fixed $n$, the numbers

$$
\lambda_{n, 0}=1, \lambda_{n, k}:=\frac{n^{k}}{(n+\alpha+\beta+2)(n+\alpha+\beta+3) \cdots(n+\alpha+\beta+k+1)}, k=1,2, \ldots,
$$

are eigenvalues of $\mathcal{B}_{n}^{\alpha, \beta}$; see [11].

Let $q_{n, k}$ be an eigenpolynomial associated with $\lambda_{n, k},\left\|q_{n, k}\right\|=1$, where $\|\cdot\|$ stands for the uniform norm on $C[0,1]$. Suppose that $\mathcal{B}_{n}^{\alpha, \beta}$ is Ulam stable with Ulam constant $K_{n}$. Since $\mathcal{B}_{n}^{\alpha, \beta}$ is injective,

$$
\left\|q_{n, k}\right\| \leq K_{n}\left\|\mathcal{B}_{n}^{\alpha, \beta} q_{n, k}\right\|, k=0,1, \ldots,
$$

i.e.,

$$
1 \leq K_{n} \lambda_{n, k}, k=0,1, \ldots
$$

However, $\lim _{k \rightarrow \infty} \lambda_{n, k}=0$, which contradicts the previous inequality. This concludes the proof of (i), and (ii) can be proven similarly.

Remark 3. Let $\alpha, \beta>0$. Then, $\mathcal{B}_{n}^{\alpha, \beta}$ has a continuous kernel; hence, it is a compact operator; see, e.g., [10] ([Theorem 8.7-5]). Consequently, according to Example 1, $\mathcal{B}_{n}^{\alpha, \beta}-$ I is Ulam stable. Theorem 3.1 shows that $\mathcal{B}_{n}^{\alpha, \beta}$ is not Ulam stable. Now, we have two Ulam stable operators, $\mathcal{B}_{n}^{\alpha, \beta}-I$ and I, for which the sum is not Ulam stable.

\section{An Example and an Open Problem}

In the definition of a $C_{0}$-semigroup of operators $\left(T_{t}\right)_{t \geq 0}$ on a Banach space $X$ (see, e.g., [13]), the composition of operators plays an essential role. Concerning the problem of Ulam stability of the operators $T_{t}$, we present an example and an open problem.

Example 2. Let $X$ be the Banach space of all bounded, uniformly continuous functions on $[0, \infty)$ with the supremum norm. For $f \in X$, define:

$$
(T(t) f)(s):=f(t+s), t \geq 0, s \geq 0 .
$$


Then, $(T(t))_{t \geq 0}$ is a $C_{0}$-semigroup of operators on $X ;$ see, e.g., [13] ([Example 1.3.7]). We will prove that each $T(t)$ is an Ulam stable operator, and its smallest Ulam constant is 1.

Let $f \in X,\|T(t) f\| \leq 1$ for a certain $t \geq 0$. Then:

$$
|f(t+s)| \leq 1, s \geq 0 .
$$

Define $\varphi \in X$ by $\varphi(u):=\left\{\begin{array}{l}f(u)-f(t), 0 \leq u \leq t, \\ 0, u \geq t .\end{array}\right.$

Then, $(T(t) \varphi)(s)=\varphi(t+s)=0, s \geq 0$, i.e., $T(f) \varphi=0$. Thus, $\varphi \in N(T(t))$. Moreover,

$$
f(u)-\varphi(u)=\left\{\begin{array}{l}
f(t), 0 \leq u \leq t, \\
f(u), u \geq t,
\end{array}\right.
$$

and (11) shows that $\|f-\varphi\| \leq 1$. Consequently, $T(t)$ is Ulam stable, with Ulam constant 1 .

Let $K$ be another Ulam constant for $T(t)$. Consider $f \in X, f(u)=1, u \in[0, \infty)$. Then, there exists $g \in N(T(t))$ such that $\|f-g\| \leq K\|T(t) f\|$. This implies $|1-g(t)| \leq K$. Since $g(t)=0$, we conclude that $K \geq 1$, and so, one is the smallest Ulam constant for $T(t)$.

Problem 1. Let $B_{n} f(x):=\sum_{k=0}^{n}\left(\begin{array}{l}n \\ k\end{array}\right) x^{k}(1-x)^{n-k} f\left(\frac{k}{n}\right), f \in C[0,1], x \in[0,1]$, be the classical Bernstein operators on $C[0,1]$. It is known (see, e.g., [14-16]) that for each $t \geq 0, f \in C[0,1]$, and for each sequence of positive integers $(k(n))_{n \geq 1}$ such that $\lim _{n \rightarrow \infty} \frac{k(n)}{n}=t$, there exists $T(t) f:=\lim _{n \rightarrow \infty} B_{n}^{k(n)} f$, uniformly on $[0,1]$. Moreover, $(T(t))_{t \geq 0}$ is a $C_{0}$-semigroup of operators on $C[0,1]$ with the supremum norm.

At the International Conference on Ulam's Type Stability, Politehnica University of Timişoara, Department of Mathematics, 8-13 October 2018, Timişoara, Romania, the second author raised the following problem:

Let $t>0$. Is $T(t)$ Ulam stable?

As far as we know, this problem is still open.

\section{Conclusions and Future Work}

Our paper addresses questions of the following type. Given the Ulam stable operators $T$ and $S$ acting on Banach spaces, what about the Ulam stability of $T S, T+S, T^{k}, T$ restricted to a closed subspace, etc.? Under suitable hypotheses, we simplify some proofs and generalize some existing results. Speaking about the composition of operators, we consider discrete semigroups and $C_{0}$-semigroups with Ulam stable or Ulam unstable members. At the end of the paper, we present an open problem. We intend to return to it, in the more general context of the Ulam stability of the operators forming a semigroup. Other possible questions in this context are: (i) Is there a $C_{0}$-semigroup $(T(t))_{t \geq 0}$ on a Banach space such that for a certain $s>0, T(s)$ is Ulam stable, and for a certain $u>0$, $T(u)$ is Ulam unstable? (ii) What about the Ulam stability of the operators $T(t)$ and the Ulam stability of the infinitesimal generator?

Author Contributions: These authors contributed equally to this work. All authors read and agreed to the published version of the manuscript.

Funding: Project financed by Lucian Blaga University of Sibiu \& Hasso Plattner Foundation research grants LBUS-IRG-2019-05.

Conflicts of Interest: The authors declare no conflict of interest. 


\section{References}

1. Brzdek, J.; Popa, D.; Raşa, I.; Xu, B. Ulam stability of operators. In Mathematical Analysis and Its Applications, 1st ed.; Academic Press: Dordrecht, The Netherlands, 2018.

2. Hirasawa, G.; Miura, T. Hyers-Ulam stability of a closed operator in a Hilbert space. Bull. Korean. Math. Soc. 2006, 43, 107-117. [CrossRef]

3. Brezis, H. Functional Analysis, Sobolev Spaces and Partial Differential Equations; Springer Science \& Business Media: Berlin/Heidelberg, Germany, 2010.

4. Brzdek, J.; Popa, D.; Raşa, I. Hyers-Ulam stability with respect to gauges. J. Math. Anal. Appl. 2017, 453, 620-628. [CrossRef]

5. Brillouet-Belluot, N.; Brzdek, J.; Cieplinski, K. On some recent developments in Ulam's type stability. Abstract Appl. Anal. 2012, 2012, 716936. [CrossRef]

6. Johnson, P.S.; Balaji, S. Hyers-Ulam stability of linear operators in Fréchet spaces. Appl. Math. Inf. Sci. 2012, $6,525-528$.

7. Miura, T.; Miyajima, S.; Takahasi, S.E. Hyers-Ulam stability of linear differential operator with constant coefficients. Math. Nachr. 2003, 258, 90-96. [CrossRef]

8. Popa, D.; Raşa, I. On the stability of some classical operators from approximation theory. Expo. Math. 2013, 31, 205-214. [CrossRef]

9. Cain, B. The kernel of the composition of two operators. J. Math. Anal. Appl. 1992, 170, 472-476. [CrossRef]

10. Kreyszig, E. Introductory Functional Analysis with Applications; Wiley: Hoboken, NJ, USA, 1978.

11. Gonska, H.; Raşa, I.; Stănilă, E.-D. Beta Operators with Jacobi Weights; Constructive Theory of Functions, Sozopol 2013; Ivanov, K., Nikolov, G., Uluchev, R., Eds.; Prof. Marin Drinov Academic Publishing House: Sofia, Bulgaria, 2014; pp. 99-112.

12. Gonska, H.; Heilmann, M.; Lupaş, A.; Raşa, I. On the Composition and Decomposition of Positive Linear Operators III: A Non-Trivial Decomposition of the Bernstein Operator; Technical Report SM-DU-739; Univ. Duisburg_Essen: Duisburg, Germany, 2011.

13. Pazy, A. Semigroups of Linear Operators and Applications to Partial Differential Equations; Springer: New York, NY, USA, 1983.

14. Altomare, F.; Campiti, M. Korovkin-Type Approximation Theory and Its Applications; Series: De Gruyter Studies in Mathematics, 17; Walter de Gruyter: Berlin, Germany, 1994.

15. Altomare, F.; Montano, M.C.; Leonessa, V.; Raşa, I. Markov Operators, Positive Semigroups and Approximation Processes, 61; De Gruyter Studies in Mathematics; Walter de Gruyter: Berlin, Germany, 2014.

16. Hodiş, S.; Mesaroş, L.; Raşa, I. Smoothness and shape preserving properties of Bernstein semigroup, Mediterr. J. Math. 2018, 15, 96.

(C) 2020 by the authors. Licensee MDPI, Basel, Switzerland. This article is an open access article distributed under the terms and conditions of the Creative Commons Attribution (CC BY) license (http:/ / creativecommons.org/licenses/by/4.0/). 